\title{
DISEÑO Y VALIDACIÓN DE UNA HERRAMIENTA PARA EVALUAR MEDIOS MULTIMEDIA MUSICALES.
}

\section{DESIGN AND VALIDATION OF AN EVALUATION TOOL FOR MUSIC MULTIMEDIA RESOURCES}

\author{
Eduard Masdeu Yélamos \\ emasdeu@umanresa.cat \\ Universitat de Vic - Universitat Central de Catalunya
}

\begin{abstract}
RESUMEN
En este artículo se presenta el diseño de una herramienta para la evaluación de medios multimedia musicales. Esta herramienta fue diseñada y validada en un trabajo de investigación que tenía como objetivo dar respuesta a las necesidades del profesorado de música cuando desea incorporar la utilización de medios multimedia en el aula. La propuesta fue sometida a un proceso de juicio de expertos. La herramienta cuenta con un apartado centrado en los datos de carácter identificativo del medio multimedia musical y 18 criterios distribuidos en tres dimensiones que determinan el nivel de calidad de los medios multimedia musicales. Al final del artículo se indican algunas pautas para la correcta utilización de la herramienta.
\end{abstract}

PALABRAS CLAVE: herramienta de evaluación, medio multimedia, educación musical, tecnología, integración curricular.

\section{ABSTRACT}

In this article, the design of an evaluation tool for music multimedia resources will be presented. This tool was developed and validated in a research project that aimed to address the needs of music teachers willing to integrate music multimedia resources in the classroom. The proposal was validated through expert judgement. The evaluation tool contains a section focused on the identification data of the music multimedia resource and 18 criteria distributed in three dimensions that determine the quality of the multimedia resource. At the end of the article, there are some guidelines for using the evaluation tool.

KEY WORDS: evaluation tool, multimedia resource, music education, technology, curricular integration. 


\section{INTRODUCCIÓN}

La inclusión de las tecnologías en la enseñanza de la música puede aportar grandes beneficios en los procesos educativos, tanto a nivel de práctica docente como de aprendizaje del alumnado. Desde esta perspectiva, muchos centros educativos están digitalizando sus aulas tradicionales de música con la incorporación de herramientas tecnológicas que, utilizadas en el momento adecuado y de manera adecuada, mejoran la calidad y la eficacia de los aprendizajes escolares. Un ejemplo de estas herramientas es el medio multimedia musical.

Entendemos por medio multimedia musical aquel recurso digital que presenta contenido musical al usuario en diversos formatos (textual, icónica-visual o icónica-sonora) y de forma no lineal (Cabero \& Duarte, 1999). Díaz (2002, p.239) añade que los medios multimedia presentan los contenidos de tal modo que entretienen y motivan al usuario, pues "la imagen, por su propia naturaleza, comunica en forma más primitiva y emotiva que la palabra". Por su parte, Marquès (2004) destaca que los medios multimedia favorecen la multisensorialidad ya que combinan información icónica y abstracta. Por último, cabe puntualizar que lo que diferencia el medio multimedia musical del medio multimedia no son sus características técnicas, sino su contenido.

En comparación con los materiales tradicionales, los medios multimedia suponen un salto cualitativo en la manera de aprovechar las posibilidades que hasta el momento habían ofrecido el lenguaje escrito, y han abierto nuevas maneras de combinar diferentes medios en un nuevo entorno con el fin de ser más eficaces y significativos en el proceso de aprendizaje, y no sólo como un adorno o complemento de la información (Ríos \& Cebrián, 2000; Martí, 1992). Siguiendo esta línea, Marquès (2004) identifica 4 ventajas que presentan los medios multimedia musicales respecto a los materiales tradicionales:

- Corrección inmediata. La retroalimentación inmediata a las respuestas y a las acciones de los usuarios permite al alumnado conocer sus errores en el momento en que se producen.

- Individualización. Permiten individualizar el trabajo de los alumnos en función de sus conocimientos previos y ritmos de aprendizaje.

- Interacción. Los estudiantes están permanentemente activos al interactuar con el ordenador y mantienen un alto grado de implicación en el trabajo.

- Motivación. La interacción con los medios multimedia motivan y mantienen el interés a los alumnos.

Ahora bien, aun teniendo en cuenta las potencialidades que pueden ofrecer los medios multimedia como herramientas de aprendizaje, es un error pensar que por el solo hecho de incorporarlos se mejora la calidad y la eficacia de los aprendizajes. En este sentido, no todos los medios existentes en el mercado actual presentan una calidad mínima necesaria para utilizarse en actividades formativas desarrolladas en el aula ni tampoco ofrecen todas las posibilidades de explotación didáctica que permiten los medios multimedia (Watkins, 2001). En este contexto, Alcantud (2000, p.9) opina que "el software educativo en particular no sufre una evaluación exhaustiva antes de ser distribuido. Así como los libros de texto en las escuelas son examinados por las autoridades educativas y homologados, no se hace ninguna acción de homologación en el software educativo, ni tan solo se evalúa su usabilidad". Atendiendo a estas consideraciones, creemos necesario que se establezcan herramientas de evaluación que 
permitan a los docentes de música resolver la problemática de cómo determinar el nivel de calidad de un medio multimedia musical.

\section{METODOLOGÍA}

\subsection{Objetivo}

Esta investigación tiene como propósito diseñar, construir y validar un instrumento de evaluación que permita a los profesores de música determinar el nivel de idoneidad de un medio multimedia musical que pretende ser incorporado en el aula de música.

\subsection{Fases de trabajo.}

En cuanto al proceso de diseño, construcción y validación de la herramienta, se establecen tres fases de trabajo:

- Primera fase: Identificación y redacción de las dimensiones y criterios. En ella se idea una propuesta inicial de herramienta a partir de la información obtenida de la revisión bibliográfica y documental de estudios previos sobre el análisis de medios multimedia (Bolaño, 2017; Cova \& Arrieta \& Aular, 2008; AET, 1988; Barroso, Medel \& Valverde, 1998; Cabero \& Duarte, 1999; Gómez, 1998; Insa \& Morata, 1998; Marquès, 1998; Martínez, Prendes, Alfageme, Amorós, Rodríguez \& Solano, 2002) y de la propia experiencia profesional en el campo de la educación musical de la tecnología educativa.

- Segunda fase: Validación del instrumento inicial. Consiste en validar la propuesta inicial de herramienta mediante la evaluación por jueces expertos.

- Tercera fase: Refinado del instrumento. Consiste en revisar y modificar la herramienta en base a las aportaciones y puntuaciones obtenidas durante el procedimiento de validación, y en realizar una prueba piloto de aplicación de la herramienta con el fin de ultimarla.

\subsection{Validación por expertos.}

La propuesta inicial de herramienta fue sometida a un proceso de juicio de expertos. En total, se seleccionaron 20 expertos en la utilización de las tecnologías aplicadas al campo de la educación musical mediante un muestreo no probabilístico intencionado (Otzen \& Manterola, 2017; Alaminos \& Castejón, 2006; McMillan \& Schumacher, 2005). Concretamente, se optó por pedir opinión a 4 grupos diferentes de jueces: 5 docentes del ámbito de Educación Primaria, 5 profesores de Educación Secundaria, 5 profesores de Conservatorio y/o Escuela de Música y 5 profesores universitarios.

A los expertos seleccionados se les solicitó que valoraran los tres bloques de la herramienta (técnica - estética, pedagógica-funcional, y musical) conforme a una escala Likert de seis niveles (Nula, Muy baja, Baja, Alta, Muy alta e Imprescindible) y en términos de importancia, pertinencia y univocidad. Además de las valoraciones anteriores, la herramienta incluía un espacio abierto para que cada experto pudiera expresar cualquier comentario que estimara oportuno acerca de cada uno de los bloques y criterios de la herramienta. Para ello, se les entregó una copia de la herramienta, una breve explicación sobre el objetivo que se pretendía conseguir con el instrumento e instrucciones sobre el procedimiento que debían seguir para valorarlo.

Una vez recogidas las valoraciones sobre la herramienta, se procedió a considerar la aceptación, o no, de las dimensiones y de los criterios de evaluación según las respuestas proporcionadas por los jueces expertos. 


\subsection{Resultados}

De los 20 expertos a los que se remitió el instrumento, se reciben 19 debidamente cumplimentados: 5 rellenados por maestros de Primaria, 5 por profesores de Secundaria, 5 por profesores de Conservatorio y/o escuelas de música y 4 por profesores de Universidad. En la consideración de las puntuaciones aportadas por los jueces en relación a las dimensiones y los criterios de evaluación, destacamos que:

- Se descartan aquellos criterios en los que el número de asignaciones a las opciones "Nula, Muy baja, Baja y Alta" es igual o mayor al 50\%, pues ello supone que la mitad o más de jueces expertos consideran que el ítem o dimensión no es relevante para la evaluación de un medio multimedia musical. Concretamente, se eliminan los criterios relativos a: Originalidad, Facilita el trabajo cooperativo, Potencialidad de los recursos e Inclusión de instrumentos electrónicos (Tabla 1).

- Se calcula el índice de pertinencia y comprensión en todas las dimensiones y criterios que no han sido eliminadas en el punto anterior. El valor obtenido se compara con una relación de valores de niveles y, en función del resultado, se mantiene o descarta la dimensión o criterio. En aplicación de éste proceso no se elimina ningún ítem (Tabla 2 y Tabla 3).

- Se contemplan los comentarios y sugerencias realizadas por los jueces expertos. Concretamente, se modifica la redacción de algunos criterios y se añade una casilla en el apartado centrado en los datos de carácter identificativo del medio multimedia musical que permita distinguir si el medio analizado es gratuito o de pago.

\begin{tabular}{|c|c|c|c|c|c|c|}
\hline \multirow[t]{2}{*}{ DIMENSIÓN / CRITERIO } & \multicolumn{6}{|c|}{ FRECUENCIAS } \\
\hline & NULA & $\begin{array}{l}\text { MUY } \\
\text { BAJA }\end{array}$ & BAJA & ALTA & $\begin{array}{l}\text { MUY } \\
\text { ALTA }\end{array}$ & IMPRESCINDIBLE \\
\hline Dimensión técnica - estética & 0 & 0 & 5 & 4 & 5 & 5 \\
\hline $\begin{array}{l}\text { Dimensión pedagógica - } \\
\text { funcional }\end{array}$ & 0 & 1 & 1 & 0 & 9 & 8 \\
\hline Dimensión musical & 0 & 0 & 2 & 0 & 5 & 12 \\
\hline \multicolumn{7}{|l|}{ DIMENSIÓN TÉCNICA - ESTÉTICA } \\
\hline Diseño de las pantallas & 0 & 0 & 1 & 5 & 5 & 8 \\
\hline Calidad del sonido & 0 & 0 & 0 & 3 & 6 & 10 \\
\hline $\begin{array}{l}\text { Legibilidad de } \quad \text { los } \\
\text { contenidos }\end{array}$ & 0 & 0 & 3 & 5 & 6 & 5 \\
\hline Estructura & 0 & 0 & 1 & 6 & 6 & 6 \\
\hline Navegación & 0 & 0 & 1 & 7 & 6 & 5 \\
\hline Facilidad de interacción & 0 & 0 & 3 & 5 & 1 & 10 \\
\hline Integración de los medios & 0 & 0 & 0 & 6 & 8 & 5 \\
\hline Originalidad & 0 & 0 & 4 & 7 & 4 & 4 \\
\hline \multicolumn{7}{|c|}{ DIMENSIÓN PEDAGÓGICA - FUNCIONAL } \\
\hline Capacidad de motivación & 0 & 0 & 3 & 2 & 8 & 6 \\
\hline $\begin{array}{l}\text { Adaptación a distintos } \\
\text { ritmos de aprendizaje }\end{array}$ & 0 & 2 & 0 & 0 & 7 & 10 \\
\hline $\begin{array}{l}\text { Facilita el trabajo } \\
\text { cooperativo }\end{array}$ & 0 & 3 & 2 & 9 & 3 & 2 \\
\hline Potencialidad de los & 0 & 1 & 0 & 10 & 7 & 1 \\
\hline
\end{tabular}




\begin{tabular}{|c|c|c|c|c|c|c|}
\hline \multicolumn{7}{|l|}{ recursos } \\
\hline $\begin{array}{l}\text { Fomento } \\
\text { autoaprendizaje }\end{array}$ & 0 & 1 & 0 & 2 & 13 & 3 \\
\hline $\begin{array}{l}\text { Realiza el feedback } \\
\text { inmediato }\end{array}$ & 0 & 1 & 1 & 7 & 2 & 8 \\
\hline Evaluación del aprendizaje & 0 & 1 & 0 & 7 & 10 & 1 \\
\hline Guía didáctica & 0 & 1 & 1 & 5 & 8 & 4 \\
\hline \multicolumn{7}{|l|}{ DIMENSIÓN MUSICAL } \\
\hline $\begin{array}{lll}\text { Calidad de } & \text { las } \\
\text { interpretaciones de } & \text { los } \\
\text { archivos sonoros } & & \\
\end{array}$ & 0 & 0 & 0 & 2 & 5 & 12 \\
\hline $\begin{array}{l}\text { Adecuación del contenido } \\
\text { musical }\end{array}$ & 0 & 0 & 3 & 3 & 4 & 9 \\
\hline $\begin{array}{l}\text { Inclusión de instrumentos } \\
\text { electrónicos }\end{array}$ & 0 & 0 & 1 & 13 & 4 & 1 \\
\hline $\begin{array}{l}\text { Secuenciación de los } \\
\text { contenidos musicales }\end{array}$ & 0 & 0 & 4 & 2 & 9 & 4 \\
\hline Calidad de los contenidos & 0 & 0 & 1 & 4 & 5 & 9 \\
\hline Cantidad de los contenidos & 0 & 0 & 3 & 5 & 10 & 1 \\
\hline
\end{tabular}

Tabla 1. Asignaciones proporcionadas por los jueces expertos.

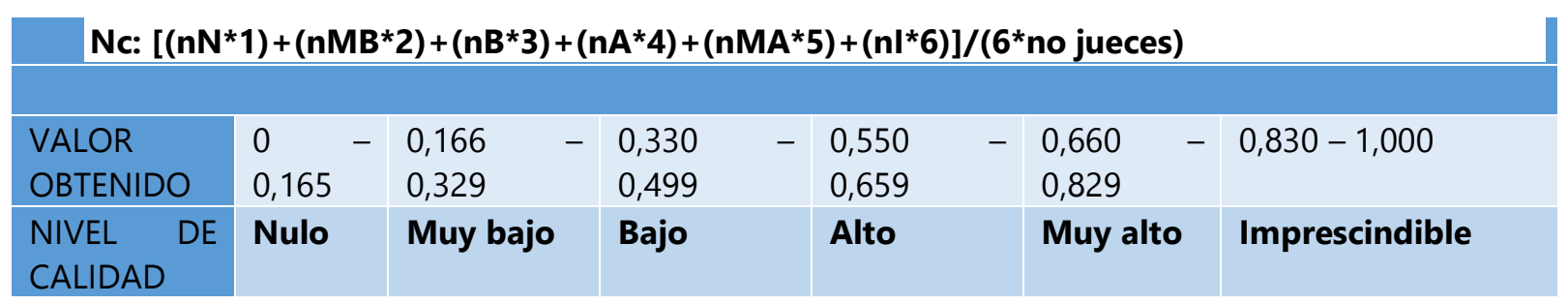

Tabla 2. Relación de niveles de calidad de los indicadores validados por jueces.

Nc: Nivel de calidad.

$\mathrm{nN}$ : Número de jueces que asignan el criterio a la opción nulo.:

nMB: Número de jueces que asignan el criterio a la opción muy bajo.

nB: Número de jueces que asignan el criterio a la opción bajo.ispep

nA: Número de jueces que asignan el criterio a la opción alto.s[s]

nMA: Número de jueces que asignan el criterio a la opción muy alto. :

nl: Número de jueces que asignan el criterio a la opción Imprescindible. [iste.

\begin{tabular}{|l|l|l|l|}
\hline DIMENSIÓN / CRITERIO & ÍNDICE & NIVEL DE CALIDAD & VALORACIÓN \\
\hline Dimensión técnica - estética & 0,754 & Muy alto & No descartado \\
\hline Dimensión pedagógica - funcional & 0,860 & Imprescindible & No descartado \\
\hline Dimensión musical & 0,904 & Imprescindible & No descartado \\
\hline Diseño de las pantallas & 0,842 & Imprescindible & No descartado \\
\hline Calidad del sonido & 0,895 & Imprescindible & No descartado \\
\hline
\end{tabular}




\begin{tabular}{|l|l|l|l|}
\hline Legibilidad de los contenidos & 0,781 & Muy alto & No descartado \\
\hline Estructura & 0,816 & Muy alto & No descartado \\
\hline Navegación & 0,798 & Muy alto & No descartado \\
\hline Facilidad de interacción & 0,825 & Muy alto & No descartado \\
\hline Integración de los medios & 0,825 & Muy alto & No descartado \\
\hline Capacidad de motivación & 0,816 & Muy alto & No descartado \\
\hline $\begin{array}{l}\text { Adaptación a distintos ritmos de } \\
\text { aprendizaje }\end{array}$ & 0,868 & Imprescindible & No descartado \\
\hline Fomento del autoaprendizaje & 0,816 & Muy alto & No descartado \\
\hline Realiza el feedback inmediato & 0,798 & Muy alto & No descartado \\
\hline Evaluación del aprendizaje & 0,754 & Muy alto & No descartado \\
\hline Guía didáctica & 0,781 & Muy alto & No descartado \\
\hline $\begin{array}{l}\text { Calidad de las interpretaciones de } \\
\text { los archivos sonoros }\end{array}$ & 0,921 & Imprescindible & No descartado \\
\hline $\begin{array}{l}\text { Adecuación del contenido musical } \\
\text { al nivel educativo }\end{array}$ & 0,833 & Imprescindible & No descartado \\
\hline $\begin{array}{l}\text { Secuenciación de los contenidos } \\
\text { musicales }\end{array}$ & 0,781 & Muy alto & No descartado \\
\hline Calidad de los contenidos & 0,860 & Imprescindible & No descartado \\
\hline Cantidad de los contenidos & 0,746 & Muy alto & No descartado \\
\hline
\end{tabular}

Tabla 3. Resultado de relación de niveles de calidad.

\section{PRESENTACIÓN DE LA HERRAMIENTA}

La herramienta de evaluación (Tabla 4) identifica 18 criterios distribuidos en tres dimensiones que determinan el nivel de calidad de los medios multimedia musicales: técnica - estética (apartado 3.1.), pedagógica-funcional (3.2.) y musical (3.3.). La herramienta cuenta con un apartado centrado en los datos de carácter identificativo del medio multimedia.

\begin{tabular}{|c|c|}
\hline \multicolumn{2}{|c|}{ Rellene los datos de carácter identificativo del medio multimedia musical a evaluar } \\
\hline \multicolumn{2}{|c|}{ HERRAMIENTA DE EVALUACIÓN DE MEDIOS MULTIMEDIA MUSICALES } \\
\hline \multicolumn{2}{|l|}{ DATOS DE CARÁCTER IDENTIFICATIVO } \\
\hline \multicolumn{2}{|l|}{ TÍTULO } \\
\hline \multicolumn{2}{|l|}{ AUTOR } \\
\hline \multicolumn{2}{|l|}{ TIPOLOGÍA } \\
\hline uSoporte físico e & disco ${ }^{(1)}$ uSoporte online ${ }^{(2)}$ \\
\hline \multicolumn{2}{|l|}{ FUNCIÓN } \\
\hline $\begin{array}{l}\text { Dlnformativa } \quad \mathrm{QE} \\
\text { QReproductora } \mathrm{Q}\end{array}$ & $\begin{array}{l}\text { valuativa GExpresiva o creativa QGuía } \\
\text { Exploradora GLúdica GEjercitación }\end{array}$ \\
\hline \multicolumn{2}{|c|}{$\begin{array}{l}\text { Responder solo si se ha seleccionado la } \text { QRequiere instalación } \square \text { No requiere instalación } \\
\text { casilla de en soporte físico (1) }\end{array}$} \\
\hline \multirow[t]{2}{*}{$\begin{array}{l}\text { Responder solo si se ha seleccionado la } \\
\text { casilla de en soporte online }\end{array}$} & QRequiere registro $\square$ QNo requiere registro \\
\hline & Enlace: \\
\hline
\end{tabular}




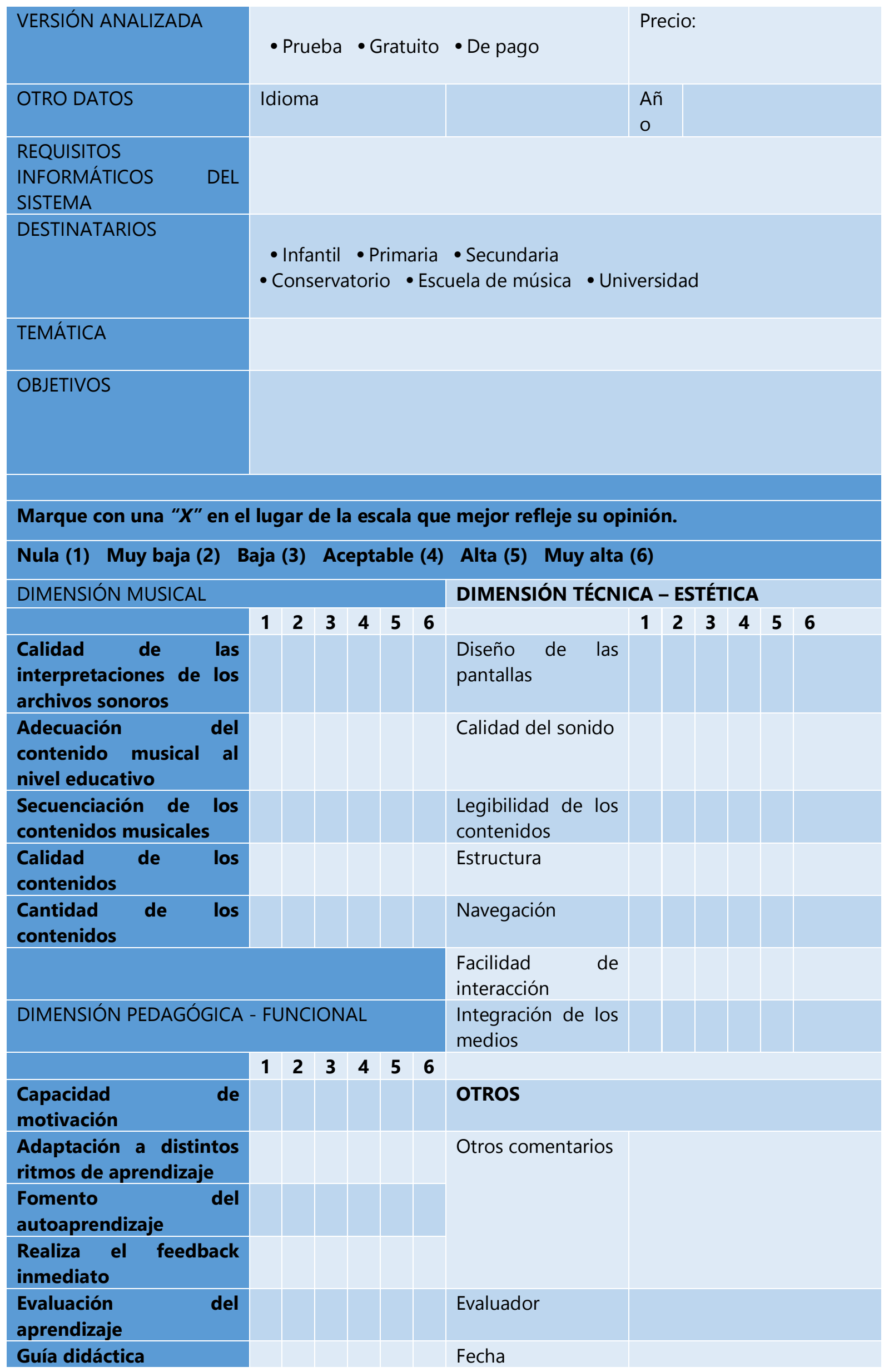


Tabla 4. Herramienta específica para la evaluación de medios multimedia musicales.

\subsection{Dimensión técnica - estética}

La dimensión técnica-estética identifica aquellos aspectos estructurales y visuales que influyen en la configuración de los medios multimedia musicales:

- Diseño de las pantallas. Forma en que el usuario va a percibir visualmente el contenido del medio multimedia musical.

- Calidad del sonido. Grado de excelencia auditiva con que el usuario va a percibir los fragmentos sonoros del medio multimedia musical.

- Legibilidad de los contenidos. Facilidad de lectura de los fragmentos de textos incluidos en el medio multimedia musical. En él, se tienen en cuenta la longitud del texto, el tamaño, el color y el tipo de letra.

- Estructura. Modo en que se agrupan las pantallas del medio multimedia musical (una página principal que enlaza a otras páginas secundarias o páginas enlazadas entre sí).

- Navegación. Elementos activos que permiten el desplazamiento entre pantallas.

- Facilidad de interacción. Grado de participación que el usuario puede establecer con el medio multimedia musical.

- Integración de los medios. Diversidad de recursos (audios, vídeos, enlaces a páginas web $u$ otros) incluidos en el medio analizado.

\subsection{Dimensión pedagógica - funcional}

La dimensión pedagógica-funcional identifica aquellos aspectos educativos y de uso que influyen en el aprendizaje de contenidos musicales:

- Capacidad de motivación. Grado de implicación e interés personal que puede suscitar en el usuario el medio multimedia musical.

- Adaptación a distintos ritmos de aprendizaje. Posibilidad del medio multimedia musical a adecuarse a las diferentes necesidades de aprendizaje del usuario.

- Fomento del autoaprendizaje. Configuración del medio multimedia musical de tal modo que el usuario pueda aprender de manera autónoma sin apoyo del profesor.

- Feedback inmediato. Capacidad del medio multimedia musical para efectuar un seguimiento al instante de las respuestas que el usuario ejecuta.

- Evaluación del aprendizaje. Información que ofrece el medio multimedia musical de los resultados obtenidos en las actividades realizadas por el usuario.

- Guía didáctica. Documento que orienta al profesor en las posibilidades de uso del medio multimedia musical.

\subsection{Dimensión musical}

La dimensión musical analiza aquellos contenidos específicos propios de la educación musical e identifica aquellos elementos que, de una u otra manera, están relacionados con elementos musicales de los medios multimedia musicales:

- Calidad de las interpretaciones de los archivos sonoros. Grado de excelencia de las actuaciones musicales incluidas en los audios del medio multimedia musical.

- Adecuación del contenido musical al nivel educativo. Capacidad de adaptación de los niveles de dificultad de las diferentes temáticas del medio en función de la etapa educativa del usuario.

- Secuenciación de los contenidos musicales. Ordenación en que se presentan las 
temáticas del medio multimedia musical.

- Calidad de los contenidos. Grado de excelencia de las temáticas incluidas en el medio.

- Cantidad de contenidos. Número de temáticas tratadas en el medio multimedia musical.

\section{PAUTAS PARA LA CORRECTA UTILIZACIÓN DE LA HERRAMIENTA}

Sugerimos unas mínimas pautas al profesorado de música para la correcta utilización de la herramienta:

- En primer lugar, el docente deberá rellenar los datos de carácter identificativo del medio multimedia musical que pretende evaluar.

- A continuación deberá evaluar la calidad del medio seleccionado. Para tal fin, dispone de 18 criterios de evaluación distribuidos en tres dimensiones (técnica - estética, pedagógica - funcional y musical) y de una tabla que incluye una escala de seis valores al lado de cada uno de los criterios (Nula, Muy baja, Baja, Aceptable, Alta y Muy alta) en los que deberá marcar con una " $X$ " la respuesta más adecuada según su punto de vista. Para cada criterio, tan sólo podrá seleccionar una única opción de las 6 disponibles.

- Al final de la herramienta se incluye un apartado (otros datos) en el que debe indicar quién ha realizado la evaluación, cuándo se ha realizado y, en caso que se quiera añadir algún otro comentario o información relacionada con el material analizado, dispone de un espacio para tal fin.

- Por último, una vez aplicada la herramienta al medio multimedia musical deseado, el docente podrá conocer qué dimensiones del medio analizado presentan una configuración global óptima para ser incorporadas dentro de un proceso de aprendizaje de calidad o cuáles, por el contrario, necesitan ser afianzadas con la presencia de otros recursos educativos por la baja calidad en alguno de sus aspectos analizados anteriormente. Para ello, el docente deberá sumar todos los resultados numéricos seleccionados de la escala de valoración de los criterios de evaluación pertinentes a una de las dimensiones de la herramienta. El valor que se obtenga de la suma se comparará con una relación de valores de niveles incluidos en la tabla (Tabla 5) y, según quede situado, se corresponderá con uno de los siguientes niveles de calidad: No recomendamos utilizar, Necesita mejorar algunos aspectos o Idóneo. Si el nivel de calidad obtenido por la dimensión es "Idóneo" recomendamos incorporar todos los aspectos del medio multimedia musical referidos a esa dimensión dentro de un proceso de aprendizaje por su óptima calidad. Si el nivel de calidad obtenido es "Necesita mejorar algunos aspectos" sugerimos que, en caso de querer incorporar los aspectos del medio referidos a esa dimensión dentro de un proceso de aprendizaje, se revisen los criterios de evaluación seleccionados en la herramienta y se consoliden aquellos aspectos del medio que hayan sido valorados con una puntuación más baja con la presencia de otros recursos educativos. Si el nivel de calidad obtenido es "No recomendamos utilizar" aconsejamos no utilizar ninguno de los aspectos del medio referidos a esa dimensión dentro de un proceso de aprendizaje debido a la deficiente calidad de sus componentes.

\begin{tabular}{l|lll|} 
& $\begin{array}{l}\text { NO RECOMENDAMOS } \\
\text { UTILIZAR }\end{array}$ & $\begin{array}{l}\text { NECESITA MEJORAR ALGUNOS } \\
\text { ASPECTOS }\end{array}$ & IDÓNEO \\
\hline $\begin{array}{l}\text { Dimensión musical } \\
\text { Dimensión }\end{array}$ & $1-10$ & $11-20$ & $21-30$ \\
$\begin{array}{l}\text { pedagógica } \\
\text { funcional }\end{array}$ & $1-12$ & $13-26$ & $27-36$ \\
\hline
\end{tabular}




\begin{tabular}{l|l|l|l} 
Dimensión técnica & $1-14$ & $15-28$ & $29-42$
\end{tabular}

Tabla 5. Relación de niveles de calidad de las dimensiones del medio analizado.

\section{CONCLUSIONES}

Los medios multimedia musicales, utilizados en el momento adecuado y de manera adecuada, pueden atender con éxito muchas deficiencias, tanto a nivel de práctica docente como de aprendizaje con el alumnado. Ahora bien, aun teniendo en cuenta las potencialidades educativas que pueden ofrecer los medios multimedia como herramientas de aprendizaje, es un error pensar que por el solo hecho de incorporarlos se mejora la calidad y la eficacia de los aprendizajes y se favorece la capacitación del alumnado en la adquisición de los conocimientos, habilidades y actitudes en el ámbito de las competencias digitales (Generalitat de Catalunya, 2010). Dicho de otra manera, la inclusión de las tecnologías es un proceso complejo en el que intervienen una serie factores que condicionan la forma en que se integran dentro del aula, de manera que tan solo su implementación tendrá un impacto significativo en los aprendizajes escolares si son incorporadas de manera reflexiva y planificada. Desde esta perspectiva, la herramienta de evaluación presentada identifica 18 indicadores -distribuidos en tres dimensiones- que determinan la calidad de un medio multimedia musical:

En la primera dimensión, técnica-estética, quedan situados aquellos aspectos estructurales y visuales que influyen en la configuración de los medios multimedia musicales: diseño de las pantallas, calidad del sonido, legibilidad de los contenidos, estructura, navegación, facilidad de interacción e integración de los medios. Si nos centramos en la dimensión pedagógicafuncional, se incluyen aquellos aspectos educativos y de uso que influyen en el aprendizaje de contenidos musicales: capacidad de motivación, adaptación a distintos ritmos de aprendizaje, fomento del autoaprendizaje, feedback inmediato, evaluación del aprendizaje y guía didáctica. En referencia a la dimensión musical, quedan situados aquellos aspectos educativos y de uso que influyen en el aprendizaje de contenidos musicales: calidad de las interpretaciones de los archivos sonoros, adecuación del contenido musical al nivel educativo, secuenciación de los contenidos musicales, calidad de los contenidos y cantidad de contenidos. La herramienta cuenta con un apartado centrado en los datos de carácter identificativo del medio multimedia. Consideramos que la herramienta presentada puede resultar interesante y de gran utilidad a todos los profesores de música que desean integrar de manera eficiente los medios multimedia musicales en la enseñanza.

Por último, como línea de investigación futura, interesa plantearse en qué medida los medios multimedia musicales posibilitan la adquisición de las competencias específicas curriculares.

\section{Referencias}

AET. (1988). Power on! New tools for teaching and Learning. Congress of the United States, Office of Technology Assessment, OTA SET-379. Recuperado de https://www.princeton.edu/ ota/disk2/1988/8831/8831.PDF

Alaminos, A. \& Castejón, J.L. (2006). Elaboración, análisis e interpretación de encuestas, cuestionarios y escalas de opinión. Alcoy: Marfil. 
Alcantud, F. (2000). Nuevas Tecnologías, Viejas Esperanzas, En F.J. Soto y J.A. López (Eds.), Nuevas Tecnologías, Viejas Esperanzas: las nuevas tecnologías en el ámbito de la discapacidad y las necesidades educativas especiales. Murcia: Consejería de Educación y Universidades.

Barroso, J. \& Medel, J. \& Valverde, J. (1998). Evaluación de medios informáticos. Una escala de evaluación para software educativo. Congreso EDUTEC. Recuperado de http://www.quadernsdigitals.net/datos/hemeroteca/r 11/nr 183/a 2316/2316.htm

Bolaño, M. (2017). Uso de Herramientas Multimedia Interactivas en educación preescolar. Didáctica, Innovación y Multimedia (DIM), 35, 1-20 Recuperado de https://ddd.uab.cat/pub/dim/dim a2017m5n35/dim a2017m5n35a4.pdf

Cabero, J. \& Duarte, A. (1999). Evaluación de medios y materiales de enseñanza en soporte multimedia. Pixel - Bit. Revista de Medios y Educación, 13, 23-45 Recuperado de http://tecnologiaedu.us.es/cuestionario/bibliovir/47.pdf

Cova, A. \& Arrieta, X. \& Aular, J. (2008). Revisión de modelos para evaluación de software educativos. Télématique. Recuperado de http://publicaciones.urbe.edu/index.php/telematique/article/viewArticle/900/2234

Generalitat de Catalunya. (2010). Educació. El pla TAC del centre. Col-lecció TAC-1. Departament d'Educació.

Recuperado

de http://ensenyament.gencat.cat/web/.content/home/departament/publicacions/collecci ons/tac/pla-tac-centre/tac 1.pdf

Gómez, M.T. (1998). Un ejemplo de evaluación de software educativo multimedia. Congreso EDUTEC.

Recuperado

de http://www.quadernsdigitals.net/index.php?accionMenu=hemeroteca.VisualizaArticulo $\underline{\text { IU.visualiza\&articulo } \mathrm{id}=2311}$

Díaz, F. (2002). Didáctica y currículo: un enfoque constructivista. Cuenca: Ediciones de la Universidad de Castilla-La Mancha.

Insa, D. \& Morata, R. (1998). Multimedia e Internet. Madrid: Paraninfo.

McMillan, J.H. \& Schumacher, S. (2005). Investigación educativa. Una introducción Conceptual. Madrid: Pearson Educación.

Marquès, P. (2004). Multimedia educativo: clasificación, funciones, ventajas e inconvenientes. Recuperado de http://peremarques.pangea.org/funcion.htm

Marquès, P. (1998). La evaluación de los programas didácticos. Comunicación y pedagogía: Nuevas tecnologías y recursos didácticos, 149, 53-58.

Martí, E. (1992). Aprender con ordenadores en la escuela. Barcelona: Horsori.

Martínez, F. \& Prendes, M.P. \& Alfageme, M.B. \& Amorós, L. \& Rodríguez, T. \& Solano, I.M. (2002). Herramienta de evaluación de multimedia didáctico. Pixel - Bit. Revista de Medios y Educación, 18, 71-88 Recuperado de https://doi.org/10.12795/pixelbit 
Otzen, T. \& Manterola, C. (2017). Técnicas de Muestreo sobre una Población a Estudio. International Journal of Morphology, 35 (1), 227-232 Recuperado de https://dx.doi.org/10.4067/S0717-95022017000100037

Ríos, J.M. \& Cebrián, M. (2000). Nuevas tecnologías de la información y la comunicación aplicadas a la educación. Málaga: Ediciones Aljibe.

Watkins, A. (2001). Aplicación de las Nuevas Tecnologías a las Necesidades Educativas Especiales. Últimas tendencias en 17 países europeos. Middelfart: European Agency for Development in Special Needs Education, 188.

Para referenciar este artículo:

Masdeu-Yélamos, E. (2018). Diseño y validación de una herramienta para evaluar medios multimedia musicales. EDUTEC, Revista Electrónica de Tecnología Educativa, 64. Recuperado de: http://dx.doi.org/10.21556/edutec.2018.64.963 